\title{
Osteoimmunomodulatory Effects of Enamel Matrix Derivate and Strontium Coating Layers: A Short- and Long-Term In Vivo Study
}

Maryam Rahmati, Matthias Johannes Frank, Sebastian Martin Walter, Marta Cabrer Monjo, Maria Satué, Janne Elin Reseland, Ståle Petter Lyngstadaas, and Håvard Jostein Haugen*

Cite This: https://dx.doi.org/10.1021/acsabm.0c00608

Read Online

ACCESS |

山ll Metrics \& More

回国 Article Recommendations

SI Supporting Information

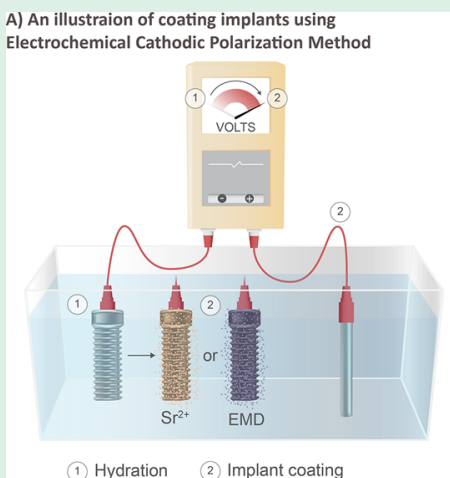

B) An illustriation of implant's functions in vivo

after coating a layer of $\mathrm{EMD}$ or $\mathrm{Sr}^{2+}$
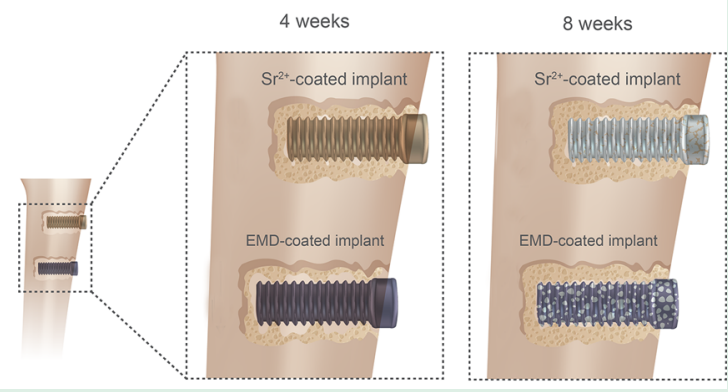

ABSTRACT: Over the past few years, surface modification of implant surfaces has gained substantial attention as a promising solution to avoid the failure of biomaterials after implantation. Although researchers suggest several strategies for surface functionalization of titanium-based implants, only a few studies have compared the osteoimmunomodulatory effects of ionic nanostructures and biofunctionalization in the same biological model. Enamel matrix derivate (EMD) and strontium are both known for their positive influences on bone cell responses. In this study, we functionalized the titanium-zirconium implant surface with EMD and strontium using an electrochemical cathodic polarization method. Afterward, we evaluated the osteoimmunomodulatory effects of EMD or strontium coated titanium-zirconium implants in the tibia of eight Gray Bastard Chinchilla rabbits. We performed 2 and 3D micro-CT, wound fluid, histologic, and histomorphometric analyses on bone tissues after 4- and 8-weeks of implantation. Although the results could indicate some differences between groups regarding the bone quality, there was no difference in bone amount or volume. EMD stimulated higher ALP activity and lower cytotoxicity in wound fluid, as well as a lower expression of inflammatory markers after 8 weeks indicating its osteoimmunomodulatory effects after implantation. Overall, the results suggested that ionic nanostructure modification and biofunctionalization might be useful in regulating the immune responses to implants.

KEYWORDS: bioactive coating, enamel matrix derivate, strontium, titanium-zirconium, biointerface, osteoimmunomodulation

\section{INTRODUCTION}

Over the past two decades, the prevalence of using bone implants has increased significantly. ${ }^{1,2}$ Despite the substantial progress in the material design, surgical implant methods, and sterile surgical principles, a large proportion of implants fail to integrate with the neighboring bone tissue after implantation. ${ }^{1,3}$ This failure causes implant encapsulation by fibrotic tissue and bacterial colonization, which causes biofilm formation on the implant surface. ${ }^{4,5}$ The fibrous encapsulation can cause poor osseointegration and implant loosening, whereas the biofilm formation is known as the main reason for surgical site infections and consequently surgical removal of the implant. ${ }^{3}$ In the United States alone, $25.6 \%$ of all healthcare-associated infections are related to devices causing a huge financial burden on both individual and public health care systems. ${ }^{6}$ Among orthopedic and dental implants, the titanium-zirconium alloy (TiZr) has gained considerable attention owing to its higher fracture toughness and corrosion resistance over other commercially available implants. ${ }^{7-9}$ Despite its benefits and high success rate over the past few years, a TiZr implant could fail in patients suffering from low bone mineral density (BMD), abnormal wound healing, or having systemic disorders like osteoporosis and diabetes. ${ }^{10,11}$

Received: $\quad$ May 22, 2020

Accepted: July 23, 2020

Published: July 23, 2020 
Owing to the costs, risks, and patient discomfort of implant failure, improving the implant surface properties is vital for these patients.

Researchers address this issue by using surface functionalization strategies, which refer to immobilizing bioactive molecules on the surface for enhancing bone-implant interactions. ${ }^{12-14}$ An optimal bioactive surface for orthopedic and dental applications must fulfill three key necessities. It must be based on convenient and effective immobilization techniques for producing materials in any size and shape. It must be strongly stable and adhered to the implant to endure the harsh implantation and daily wear conditions. It should also improve early osseointegration through enhancing the physicochemical properties of the implant surface and/or directing the biological pathways involved in host responses. ${ }^{15,16}$ With these principles in mind, many research groups investigate the bone regeneration and immunomodulatory potential of numerous bioactive molecules including growth factors, peptides, antibiotics, antiseptics, polysaccharides, and ions. ${ }^{16-18}$ The interactions between these molecules with the stem- and osteoblastic-progenitor cells determine the boneimplant bioactivity. ${ }^{19}$ Bioactive molecules can enhance osteoblast differentiation and bone healing at the interface, leading to improved bone healing. ${ }^{20,21}$ Several studies suggest immobilizing growth factors on the surface for improving cellimplant interactions. ${ }^{18,22}$ However, their safety concerns and high costs encouraged researchers to investigate the potential of using other small molecules such as ions and bone matrix extracts to simplify the therapeutic signaling. ${ }^{19,23}$

Over the past few years, the incorporation of ions (such as silver, copper, and strontium) on the implant surface has gained much attention among researchers as a simple surface modification strategy for promoting bone healing. ${ }^{24-27}$ Compared to growth factors, ions are cheaper as well as have greater simplicity, stability, and effectiveness. ${ }^{19}$ Some studies revealed that strontium ions could significantly induce bone regeneration by triggering preosteoblast proliferation as well as osteoblast differentiation and survival. ${ }^{26-28}$ Strontium ions could also prevent bone resorption through reducing preosteoclast differentiation. ${ }^{28}$ However, because the ion functions are dose-dependent, choosing the right concentrations as well as evaluating their potential short- and longterm effects in vivo are vital. ${ }^{29}$

In addition to ions, bone protein extracts such as enamel matrix derivative (EMD) from bone can be beneficial in stimulating bone formation at implant surface. ${ }^{30}$ EMD is extracted from developing porcine teeth and consists primarily of hydrophobic enamel matrix proteins including amelogenin (90\%) and other nonamelogenin enamel matrix proteins (10\%). ${ }^{31}$ Many in vitro studies demonstrated that EMD could enhance bone regeneration through stimulating differentiation of osteogenic precursors, osteogenic differentiation of periodontal ligament cells, and activation of endothelial cells. ${ }^{32-36}$ These results indicate that EMD may be a good candidate for biofunctionalization of implant surface. Takeda et al. ${ }^{31}$ investigated the EMD potential in improving periodontal tissue regeneration in diabetic conditions. They reported that EMD could enhance bone regeneration through Akt/VEGF signaling in diabetic rats. ${ }^{31}$ Despite its bone regeneration potential, EMD has some difficulties such as animal sources, immunogenicity, regulatory assessments, ethical issues, large molecular weight (100-200 amino acids), and instability in vivo. $^{37,38}$ In this study, we compared the osteoimmunomodu- latory potential of EMD with strontium ions under the same in vivo conditions to investigate whether it is worth to coat a layer of EMD with the mentioned difficulties for inducing bone regeneration or we can consider using other alternatives such as ions as a simpler and cheaper surface modification strategy.

Even though many types of biomolecules are suggested for surface functionalization, only few studies have compared the efficacy of different biomolecules with each other. ${ }^{39-41}$ To the best of our knowledge, there is no study comparing the osteoimmunomodulatory effects of EMD with strontium ions.

In our previous in vitro studies, we coated TiZr surfaces with EMD and strontium by using electrochemical cathodic polarization method and evaluated the osteoblast cell responses to the surfaces. ${ }^{42,43}$ Electrochemical polarization is a relatively simple and industrial applicable, which can be used for implant surface modification while maintaining implant mechanical properties. ${ }^{44}$ In addition, it can control biomolecules sustained release at optimal concentrations over time. ${ }^{44}$ Anodic and cathodic polarization are the two most common electrochemical techniques used for this purpose, of which both are useful for molecule deposition on the surface. ${ }^{42,45}$ Since cathodic polarization does not require high temperatures during the process, the biomolecule adhesion and coating layer can be controlled more easily and less harmful than other techniques. $^{46}$ Although some in vitro studies, including ours, ${ }^{14,42,43}$ reported that this method could be beneficial for promoting the molecule deposition on the surface, only a few in vivo studies have studied the biomolecule release manner (deposited on the surface by this method) and the following bone tissue responses in complex body conditions. ${ }^{14,42,43}$ Our in vitro results indicated that by using this method both EMD and strontium ions can be successfully deposited on the TiZr implant surfaces. ${ }^{42}$

Even though many types of strategies are suggested for surface functionalization, only a few studies have compared the efficacy of different functionalized surfaces with each other. $^{39-41}$ In this study, we compared the immune system and bone tissue responses to TiZr implants modified with strontium ions and EMD under the same experimental conditions after 4- and 8-weeks of implantation in the rabbit tibia.

\section{MATERIALS AND METHODS}

2.1. Materials. Coin shaped implants with a diameter of $6.25 \mathrm{~mm}$ and a height of $2 \mathrm{~mm}$ were used. All implants were comparable to the commercially available SLActive surface (Institut Straumann AG, Basel, Switzerland), and their properties can be found in our previous papers. ${ }^{47,48}$

2.2. Coating Procedure. After cathodic reduction of $\mathrm{TiZr}$ implants (TiZr-H), we performed the electrocoating of surfaces with EMD and strontium on a custom-made polarization setup, based on LabVIEW (National Instruments, Austin, TX, USA) hardware and software. The setup allowed individual control and monitoring of the implant's current density on eight channels. The coating of TiZr implants was done for $75 \mathrm{~min}$, while the current density was set to $0.54 \mathrm{~mA} / \mathrm{cm}^{2}$. A feedback loop with a proportional-integralderivative (PID) controller was used to maintain a constant current output throughout the whole coating.

All implants were washed with reverse osmosis deionized water in an ultrasonic bath for $5 \mathrm{~min}$ prior to electro-coating process. After washing, the implants were mounted on individual titanium holders with polytetrafluoroethylene (PTFE) caps to protect the interface between the holder and the implant from the electrolyte. The implants were kept wet in deionized water at all time during mounting to avoid carbon contamination of the surface. Electrocoating of 
implants with strontium (TiZr-Sr) was done in a buffer made of 0.25 $M$ ultrapure (99.995\% trace element free) strontium-acetate and acetic acid at $\mathrm{pH} 5$ with additional $0.1 \mathrm{M} \mathrm{NaF}$ and $1 \% \mathrm{NaCl}$ in the buffer, optimized in our previous studies. ${ }^{42,43}$

Electrocoating of implants with EMD (TiZr-EMD) was done in a buffer made of $2 \mathrm{M}$ sodium acetate and acetic acid at $\mathrm{pH} 3$. A total of $20 \mathrm{mg}$ dry stored EMD (Institut Straumann AG, Basel, Switzerland) was dissolved in $0.1 \%$ acetic acid $(2 \mathrm{~mL})$ at $4{ }^{\circ} \mathrm{C}$ and added to the buffer to a final concentration of $0.01 \mathrm{mg} / \mathrm{mL}$ for coating, optimized in our previous study. ${ }^{42}$ An additional control group (TiZr-H) was polarized only in a buffer made of $2 \mathrm{M}$ sodium acetate and acetic acid at $\mathrm{pH}$ 3. Polarization was performed at $21{ }^{\circ} \mathrm{C}$ for all treatments (Video S1). After the coating process, the implants were rinsed in deionized water for $10 \mathrm{~s}$ and dried in a protective nitrogen atmosphere to avoid surface contamination. Implants were stored dry in glass vials under nitrogen cover gas until they were placed in rabbits. The coating characterization results for both EMD and strontium can be found in our previous papers; ${ }^{42,43}$ here we only provide the EMD release data obtained by using dot blot.

2.3. Quantitation of EMD on Implants Using Immune Dot Blot. We confirmed the integrity of EMD after using matrix-assisted laser desorption/ionization (MALDI) in our previous study. ${ }^{42}$ Immune dot blot was used to quantify the amount of EMD on each implant. EMD was eluted off the surfaces using $1 \mathrm{M} \mathrm{NaOH}(0.5$ $\mathrm{mL}$ ) for $24 \mathrm{~h}$ at $8{ }^{\circ} \mathrm{C}$.

EMD diluted 1:5 from 5000 to $5 \mathrm{ng} / \mathrm{mL}$ in $0.1 \%$ acetic acid was used as the reference curve for quantification. Every sample $(5 \mu \mathrm{L})$ was applied twice on DEPC membranes (Bio-Rad Laboratories Ltd., Herts, UK); in a Millipore SNAP i.d. protein detection system (Millipore Corporation, Billercia, MA, USA). Tris-buffered saline (TBS) containing $0.1 \%$ Tween-20 (TBST) was used as a blank negative control sample. The membrane was blocked with TBS and $0.1 \%$ casein for $1 \mathrm{~h}$. The primary EMD-antibody (sheep-antiEMD; Mölnlycke Health Care AB, Gothenburg, Sweden; 1:100 in TBS) was applied for the test, while the antibody control was kept in pure TBS for $1 \mathrm{~h}$. Both membranes were washed three times in TBST for $3 \mathrm{~min}$. The secondary EMD-antibody (rabbit antisheep HPPOX; Southern Biotech, Birmingham, AL, USA) (1:1000 in TBS) and applied to both groups for $1 \mathrm{~h}$. After three washings in TBST for $3 \mathrm{~min}$ and one in deionized water for another $3 \mathrm{~min}, 3$,3-diaminobenzidine-based (DAB, Sigma-Aldrich, St. Louis, MO, USA) horseradish peroxidase reaction product $\left(0.3 \mathrm{~g} / \mathrm{L} \mathrm{DAB}, 0.03 \% \mathrm{H}_{2} \mathrm{O}_{2}\right.$, and $0.03 \% \mathrm{NiCl}_{2}$ in 50 $\left.\mathrm{mM}\left(\mathrm{NH}_{4}\right) \mathrm{HCO}_{3}\right)$ was used to stain the EMD proteins. A Kodak Gel Logic 212 imaging system (Eastman Kodak Company, Rochester, NY, USA) was used to examine the dot blot and to assess the concentration of EMD of each sample by the intensity of staining relative to the blotted standard.

The specific reaction of the primary antibody in the TiZr-EMD groups (Figure S1, lower panel) confirmed that EMD was on the surface in measurable amounts. This was in agreement with the results from MALDI presented in our previous in vitro study. ${ }^{42}$ By comparing the obtained results from coated and EMD standard samples, we observed an average of $77.0 \pm 19.2 \mathrm{ng}$ EMD per TiZr-EMD implant surface.

2.4. Rabbit Animal Model and Implantation Procedure. The chosen noncritical size defect animal model was based on two previous studies on titanium and collagen scaffolds ${ }^{49,50}$ and is a modification of a well-established rabbit tibial model. ${ }^{51,52}$

We did the surgery on eight Gray Bastard Chinchilla rabbits (6 months old, weight $2.86 \mathrm{~kg}$, Charles River Laboratories International, Inc., Research Models and Services, Sulzfeld, Germany). The Norwegian Animal Research Authority (NARA) approved and recorded the animal study. The procedures were done based on the Animal Welfare Act of June 1 2010, No 94, and Regulation on Animal Experimentation of January 15, 1996. The details of procedure can be found in our previous paper (Figure. 1). ${ }^{48} \mathrm{We}$ used group implants per group per time point (TiZr, TiZr-H, TiZr-Sr, and TiZr-EMD) to evaluate bone regeneration and immune system responses 4 and 8 weeks after the implantation surgery. Two defects were made per leg
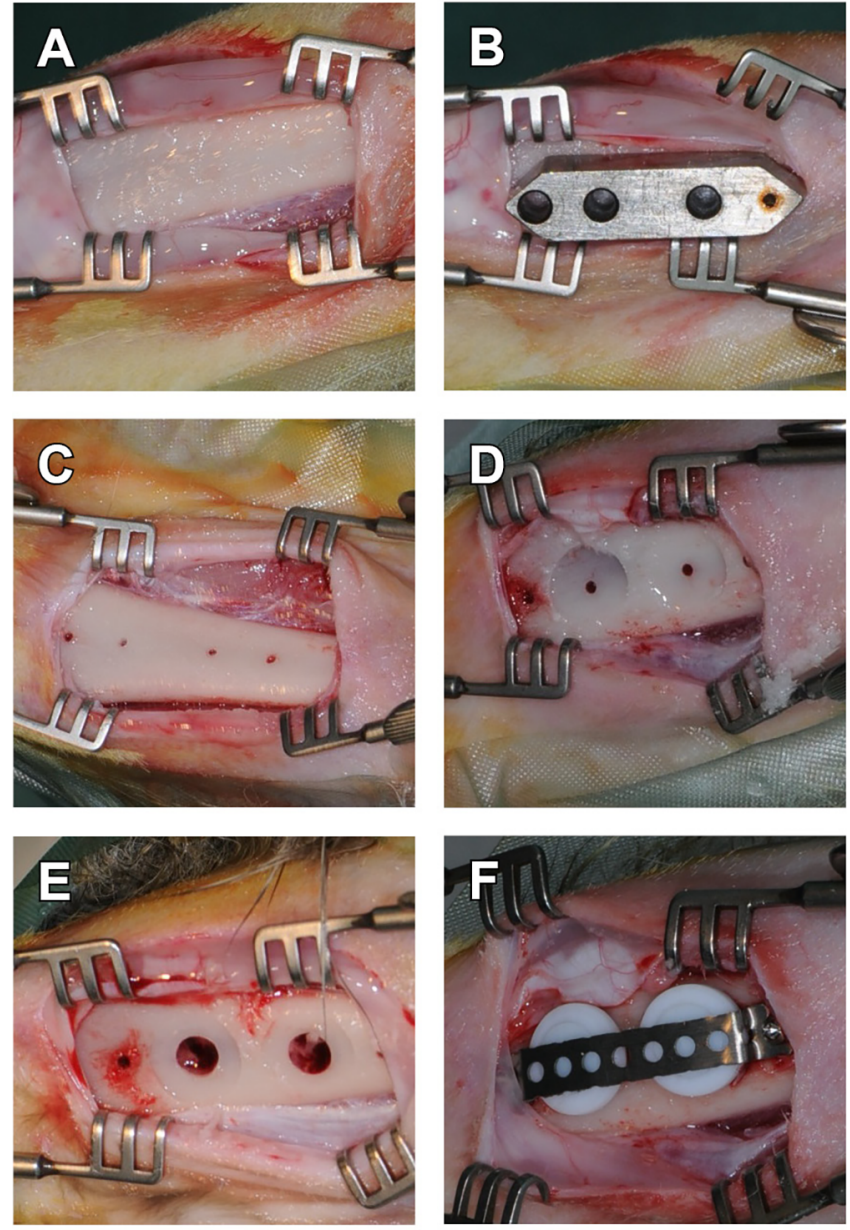

Figure 1. Illustration of the surgical procedure. After exposing the tibia (A), a hole was drilled at one end of the opening and a custommade drilling guide was fixed to the hole by a guidance pin (B). The guide was used to drill three additional holes, two for the central platforms and defects and one hole at the opposing end for retaining the titanium band $(\mathrm{C})$. The central holes were used for creating leveled platforms for coin shaped implants (D) and thereafter drilling the noncritical defects of $3.5 \mathrm{~mm}$ in diameter (E). The implants inside the protective PTFE caps were placed on the platforms and retained by a preformed maxillofacial titanium bone plate that was fixed by a titanium screw at each end $(F)$.

in each animal. Table 1 shows the distribution of implants per animals per time point.

Table 1. Distribution of Implants per Animals Per Time Point $^{a}$

\begin{tabular}{|c|c|c|c|}
\hline $\begin{array}{l}\text { rabbit } \\
\text { number }\end{array}$ & $\begin{array}{l}\text { treatment for right leg } \\
\text { defects }\end{array}$ & $\begin{array}{l}\text { treatment for left leg } \\
\text { defects }\end{array}$ & $\begin{array}{l}\text { time } \\
\text { point }\end{array}$ \\
\hline 1 & TiZr-EMD $(n=2)$ & $\operatorname{TiZr}(n=2)$ & 4 weeks \\
\hline 2 & TiZr-EMD $(n=2)$ & TiZr-H $(n=2)$ & 4 weeks \\
\hline 3 & $\operatorname{TiZr}-\mathrm{Sr}(n=2)$ & $\operatorname{TiZr}(n=2)$ & 4 weeks \\
\hline 4 & $\operatorname{TiZr}-\mathrm{Sr}(n=2)$ & TiZr-H $(n=2)$ & 4 weeks \\
\hline 5 & TiZr-EMD $(n=2)$ & $\operatorname{TiZr}(n=2)$ & 8 weeks \\
\hline 6 & TiZr-EMD $(n=2)$ & TiZr-H $(n=2)$ & 8 weeks \\
\hline 7 & $\operatorname{TiZr}-\mathrm{Sr}(n=2)$ & $\operatorname{TiZr}(n=2)$ & 8 weeks \\
\hline 8 & TiZr-Sr $(n=2)$ & TiZr-H $(n=2)$ & 8 weeks \\
\hline
\end{tabular}

${ }^{a}$ Please note that two defects were made per leg in each animal and both defects in each leg were treated with the same implant group. 

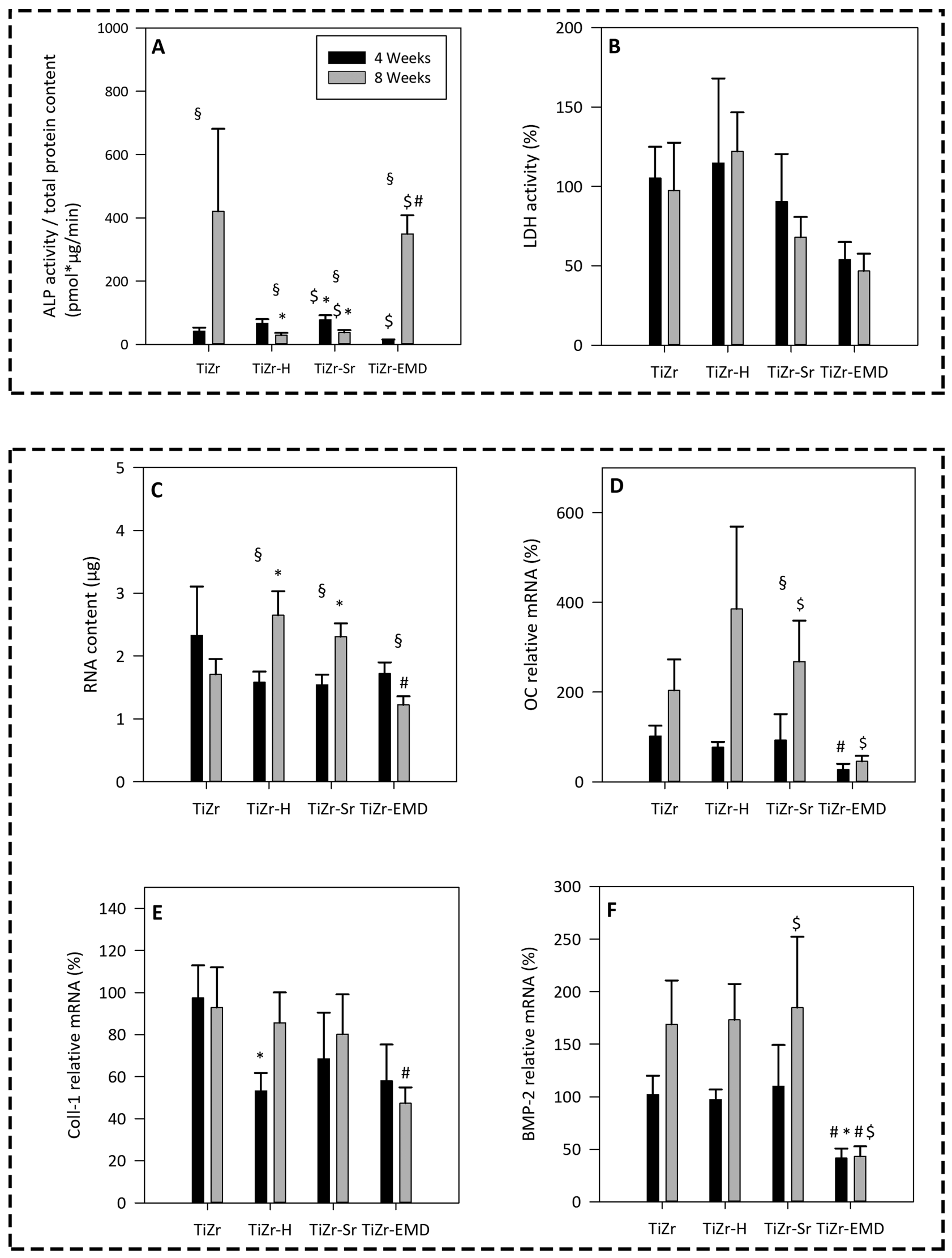

Figure 2. Analysis of wound fluid from implantation sites after implant removal (A and B) and of mRNA levels of bone formation markers in the peri-implant tissue after 4 and 8 weeks of healing. ALP (A) and LDH (B) activity in wound fluid. Total RNA content (C) and the gene level expression of bone formation markers osteocalcin (D), collagen type I (E), and bone morphogenetic protein 2 (F). Values represent the mean \pm standard deviation. Significant differences $(p<0.05)$ between groups were marked as follows: TiZr vs coated groups $(*)$, TiZr-H vs coated groups (\#), TiZr-Sr vs TiZr- EMD group (\$), and 4 vs 8 weeks of healing within a group $(\S)(n=4)$. 
After 4 and 8 weeks, the animals were euthanized using fluanison/ fentanyl (Hypnorm, Janssen Healthcare, Titusville, NJ, USA) $1.0 \mathrm{~mL}$ i.v. followed by pentobarbital (Mebumal, Rikshospitalets Apotek, Oslo, Norway), $1 \mathrm{~mL} / \mathrm{kg}$ bodyweight i.v. Immediately after euthanization an incision was made through the soft tissue on the tibia bone. The peri-implant bone tissue attached to the implants was transferred to micro centrifuge tubes containing Tripure for RNA extraction, and bone tissues were kept in $4 \%$ neutral buffered formaldehyde.

2.5. Wound Fluid Analyses. The wound fluid was collected from the site after implant detachment, using two precut Watman three filter papers. The filter papers were applied for one minute to the proximal and distal wound site for absorbing the wound fluid. Then, the papers were transferred to micro centrifuge tubes and stored at $-80^{\circ} \mathrm{C}$. Prior to analyses, the content on the filters was eluted in 200 $\mu \mathrm{L}$ and placed on ice until analyzing them on the same day.

The total protein content in the wound fluid was quantified using a bicinchoninic acid (BCA) protein assay kit (Pierce, Rockford, IL, USA) following the manufacturer's kit instructions.

Lactate dehydrogenase (LDH) activity in the wound fluid was determined spectrophotometrically after $30 \mathrm{~min}$ incubation of wound fluid $(50 \mu \mathrm{L})$ and reaction mixture $(50 \mu \mathrm{L})$ at $25^{\circ} \mathrm{C}$. The reduced nicotinamide adenine dinucleotide $(\mathrm{NADH})$ oxidation was measured at $490 \mathrm{~nm}$ in the presence of pyruvate, according to the manufacturer's kit instructions (Cytotoxicity Detection kit, Roche Diagnostics, Manheim, Germany). The absorbance was read using a micro plate reader (Asys Expert 96; Asys Hitech, Eugendorf, Austria) at $450 \mathrm{~nm}$.

The amount of ALP in the wound fluid was examined using the $p$ Nitrophenyl Phosphate (pNPP) Liquid Substrate System (SigmaAldrich, St. Louis, MO, USA). A standard curve was made by the use of Calf intestinal ALP (Promega, Madison, WI, USA). The absorbance was measured spectrophotometrically at $405 \mathrm{~nm}(\mathrm{EL} \times$ 800 Absorbance Reader, BioTek instruments, Winooski, VT, USA) and presented relative to untreated cells.

2.6. RNA Isolation and Real Time Reverse Transcription Polymerase Chain Reaction (RT-PCR) Analysis. Total RNA of the tissues attached to the implants were isolated using Tripure (Roche Diagnostics, Mannheim, Germany), according to the manufacturer's protocol. The amount was quantified at $260 \mathrm{~nm}$ using a nanodrop spectrophotometer (NanoDrop Technologies, Wilmington, DE, USA). cDNA was generated at $42{ }^{\circ} \mathrm{C}$ for $60 \mathrm{~min}$ using the high capacity RNA-to-cDNA kit (Applied Biosystems, Foster City, CA, USA)

The real-time reverse transcription-polymerase chain reaction (RTPCR) was performed in a Lightcycler 480 (Roche Diagnostics) using SYBR green detection for three housekeeping genes (18S rRNA (18S $r R N A)$, glyceraldehyde-3-phosphate dehydrogenase (GAPDH), and $\beta$ actin) and nine target genes including (bone morphogenetic protein 2 (BMP-2), collagen type I (Coll-1), osteocalcin (OC), vacuolar type proton ATPase $\left(H^{+}-A T P\right)$, tartrate-resistant acid phosphatase (TRAP), calcitonin receptor (calc-R), tumor necrosis factor- $\alpha$ (TNF- $\alpha)$, interleukin-6 (IL-6), and $I L-10)$. Primer sequences can be found in our previous study. ${ }^{53}$ Each reaction contained $7 \mu \mathrm{L}$ Lightcycler FastStart DNA MasterPLUS SYBR Green I, $0.5 \mu \mathrm{M}$ of each sense and antisense specific primers (as described by Monjo et al. ${ }^{54}$ ) and $3 \mu \mathrm{L}$ of diluted cDNA in a final volume of $10 \mu \mathrm{L}$. The amplification program included a preincubation step for the template cDNA denaturation $\left(10 \mathrm{~min}, 95^{\circ} \mathrm{C}\right)$, followed by 45 cycles of a denaturation step $(10 \mathrm{~s}$, $\left.95{ }^{\circ} \mathrm{C}\right)$, an annealing step $\left(8-10 \mathrm{~s}, 60^{\circ} \mathrm{C}\right)$, and an extension step $(10$ s, $\left.72{ }^{\circ} \mathrm{C}\right)$. After each cycle, the fluorescence was measured at $72{ }^{\circ} \mathrm{C}$ $(\lambda$ ex $470 \mathrm{~nm}, \lambda$ em $530 \mathrm{~nm}$ ). A negative control without cDNA template was run in each assay. The real time efficiencies were calculated from the obtained slopes in the LightCycler 480 software using serial dilutions. The RT-PCR products were subjected to a melting curve analysis on the LightCycler and subsequently $2 \%$ agarose/Tris-acetate-EDTA gel electrophoresis to confirm the amplification specificity, Tm and amplicon size, respectively. The target gene expression was normalized by the mean of $\beta$-actin, $18 \mathrm{~S}$ $r R N A$, and GADPH. Data for each of the target genes in the isolated samples from the modified TiZr implants were presented in percentage of the expression levels on unmodified TiZr surfaces (set to $100 \%$ ) after 4 weeks of healing.

2.7. Micro-CT Analysis. The implants were scanned using a desktop micro-CT scanner (Skyscan 1172, Bruker microCT, Kontich, Belgium); details of the scanning and image processing can be found elsewhere. ${ }^{14}$ In general, the same region of interest (ROI) was chosen for all samples (a cylinder with a diameter of $3.5 \mathrm{~mm}$ and a height of $2.5 \mathrm{~mm}$ ).

2.8. Histologic and Histomorphometric Analyses. After dehydration in a graded series of ethanol (70-100\%), the tissues were embedded in Technovit 7200VLC (Kulzer:EXAKT, Kulzer \& Co GmbH, Germany). Details of the experiment can be found in our previous paper. ${ }^{48}$

Images of all the tibia tissues were done with $10 \times$ magnification using AxioScan Z1 (Carl Zeiss, Carl Zeiss AG, Germany). The proximal and distal end sides were defined for each slide, and images were taken by focusing on the defect area with an overlapping technique of the two-implant sites. The overlapping images were examined using two parameters, horizontal (index A) and vertical (index C) dimensions of regenerated peri-implant cortical bone. ${ }^{55}$

Index A: the horizontal dimension of regenerated bone.

Index C: the vertical dimension of new peri-implant cortical bone.

The Histomorphometric parameters have been described in detail in our previous paper. ${ }^{48}$

2.9. Statistical Analysis. We did a Kolmogorov-Smirnov test to check the parametric or nonparametric distributions of the data set. A normality test was also performed (Holm-Sidak method). When the data were distributed normally, the data were presented as arithmetic mean values with standard deviation; and as median values with interquartile range when the data were not distributed normally. Twoway ANOVA on ranks were performed when the normality test failed, using the Kruskal-Wallis test for posthoc comparison; otherwise, regular ANOVA was performedwith a Tukey test for posthoc comparison. All analysis were performed in SigmaPlot 14 (Systat Software, San José, CA, USA). Significance differences were presented as significant $*_{p}<0.05$, and highly significant differences were presented as $* * p<0.01$.

\section{RESULTS}

3.1. Total Protein Content, ALP, and LDH Activity in Wound Fluid. The total protein content results indicated that ALP activity was significantly higher for TiZr-Sr group after 4 weeks and significantly lower after 8 weeks of healing compared to other groups (Figure 2A). In addition, compared to TiZr-H group, the ALP activity of the TiZr-EMD group was significantly lower after 4 weeks and significantly higher after 8 weeks of healing. $\mathrm{LDH}$ activity in wound fluid was lower in the TiZr-EMD group at both time points compared to other groups; however, differences were not significant statistically (Figure 2B).

The total RNA content from the bone tissue attached to the implants was significantly different at 8 weeks compared to 4 weeks for all treated groups (Figure 2C). TiZr-H and TiZr-Sr groups had significantly higher total RNA content than the TiZr group after 8 weeks of healing. In the TiZr-EMD group, the amount of RNA decreased after 8 weeks compared to 4 weeks of healing, and it was significantly lower than that of the TiZr-Sr group.

3.2. Bone and Inflammatory Markers in the Periimplant Tissue. Analyzing bone formation related markers (OC, BMP-2, and Coll-1) exhibited a typical gene expression profile during wound healing, with a trend toward increased expression over time (Figure 2D-F). However, the TiZr-Sr group exhibited significantly higher $O C$ after 8 weeks of 

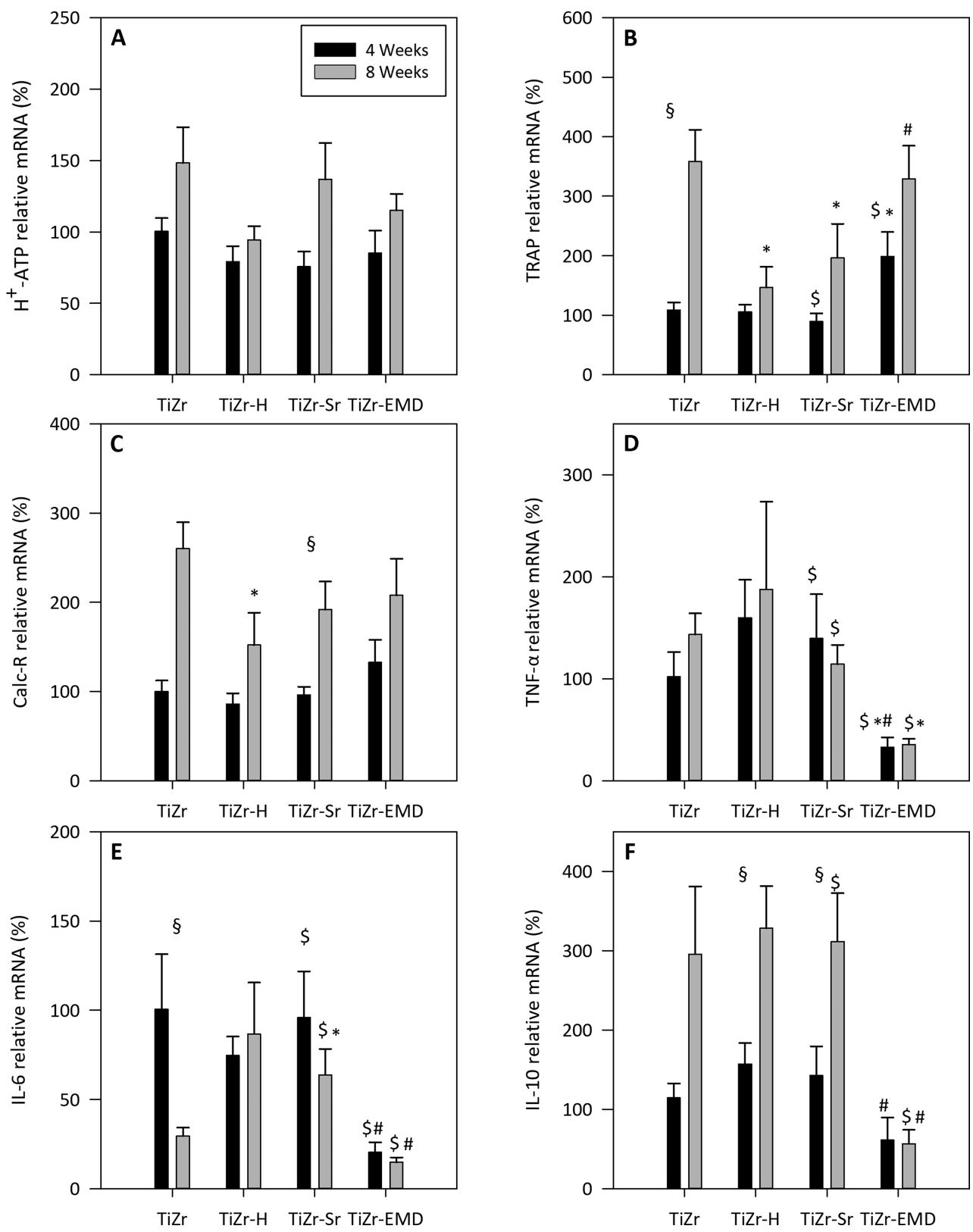

Figure 3. Expression of bone resorption related markers H+-ARP (A), TRAP (B), and Calc-R (C) as well as inflammatory markers TNF- $\alpha$ (D), IL$6(\mathrm{E})$, and IL-10 (F). Significant differences $(p<0.05)$ between groups were marked as follows: TiZr vs coated groups $(*)$, TiZr-H vs coated groups (\#), TiZr-Sr vs TiZr- EMD group $(\$)$, and 4 vs 8 weeks of healing within a group $(\$)(n=4)$.

healing. $O C$ and BMP-2 showed lower gene expression in the TiZr-EMD group after 8 weeks of healing, whereas the Coll-1 gene expression was not different from the TiZr-Sr group.

The expression of bone resorption markers $\left(H^{+}-A T P, T R A P\right.$, and $(a l c-R)$ was significantly lower in the TiZr-H and TiZr-Sr groups compared to that of TiZr and TiZr-EMD after 8 weeks (Figure $3 \mathrm{~A}-\mathrm{C}$ ). In TiZr group, there was a significantly higher expression of TRAP and Calc-R from 4 to 8 weeks of healing. The TiZr-Sr group exhibited a higher expression of Calc- $R$ after 8 weeks compared to 4 weeks of healing. Regarding the inflammatory markers (TNF- $\alpha, I L-6$, and $I L-10)$, there was a significantly lower expression of all markers for the TiZr-EMD group compared to other groups (Figure 3D). However, the TiZr-Sr group had a significantly higher expression of $I L-6$ than that of TiZr after 8 weeks of healing. In addition, both TiZr-Sr and TiZr-EMD groups exhibited a significantly lower expression of inflammatory markers after 8 weeks of healing (Figure $3 \mathrm{E}$ and $\mathrm{F}$ ).

3.3. Micro-Computed Tomography Analysis. The cross-sectional 3D images of tissues after 4 weeks of implantation demonstrated less bone healing around the defect site in TiZr, TiZr-H and TiZr-EMD groups compared to the TiZr-Sr group (Figure 4A). However, TiZr-EMD group stimulated more bone regeneration than other groups after 8 weeks of healing (Figure 4B). Figure 4C exhibits the 3D morphometric analysis of bone structure parameters (bone volume, bone surface to volume ratio, bone mineral density (BMD), and bone surface/tissue volume). After 4 weeks of healing, all bone parameters demonstrated higher values in TiZr-H and TiZr-Sr groups compared to TiZr and TiZr-EMD groups. However, after 8 weeks of healing, bone volume, BMD, and bone surface/tissue volume parameters were higher in 

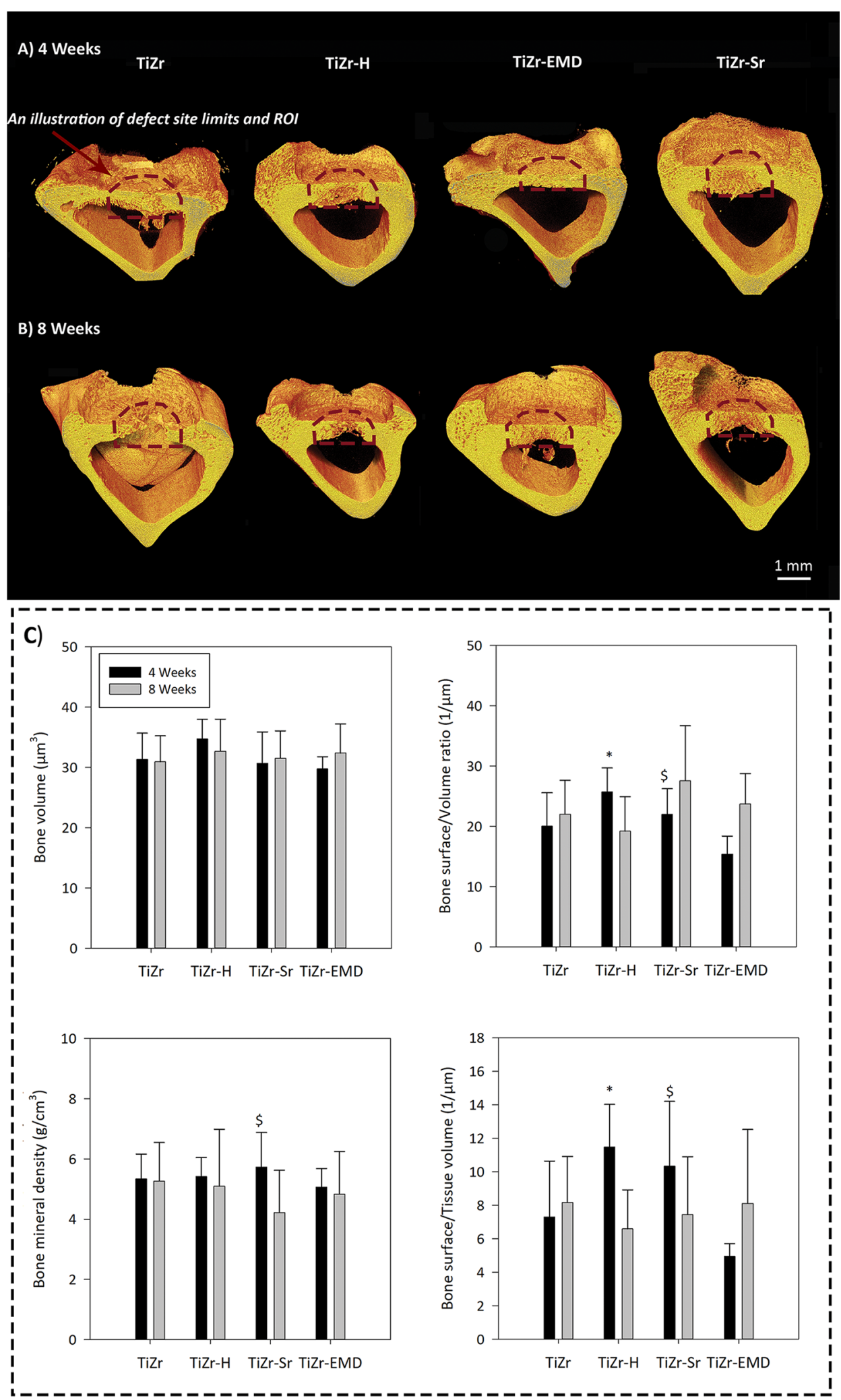

Figure 4. Cross-sectional 3D micro-CT analysis of tibia tissues. Representative cross-sectional 3D micro-CT images of tibia tissues 4 (A) and 8 (B) weeks after the implantation surgery. (C) 3D micro-CT analysis of bone volume $\left(\mu \mathrm{m}^{3}\right)$, bone surface to volume ratio $(1 / \mu \mathrm{m})$, bone mineral density $\left(\mathrm{g} / \mathrm{cm}^{3}\right)$, and bone surface/tissue volume $(1 / \mu \mathrm{m})$ for all implant groups. Values represent the mean \pm standard deviation. Significant differences $(p$ $<0.05)$ among the groups were marked as follows: ${ }^{*}=$ compared to TiZr at the same healing time; $\$=\mathrm{TiZr}-\mathrm{Sr}$ compared to TiZr-EMD at the same healing time $(n=4)$.

TiZr-EMD group compared to TiZr-Sr and comparable to TiZr-H group. After comparing the differences in bone structure parameters between 4 weeks and 8 weeks of healing, TiZr-EMD group demonstrated a positive temporal develop- 


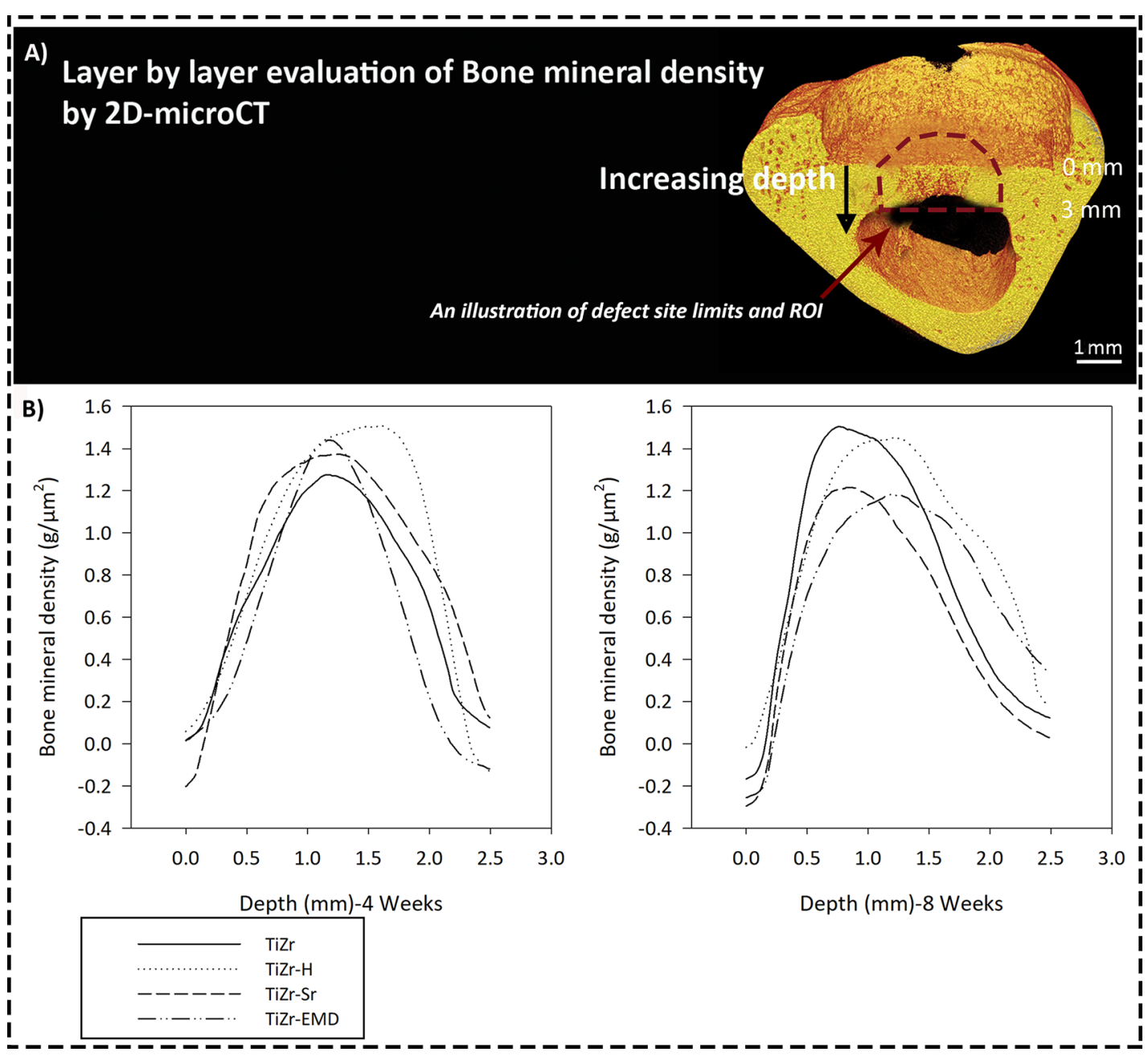

Figure 5. (A) Representative micro-CT image of implanted bone tissue showing the layer by layer evaluation of bone mineral density (BMD) using 2D micro-CT. (B) Depth profiles of bone mineral density within a circular plane $3.5 \mathrm{~mm}$ in diameter and $0.008 \mathrm{~mm}$ in height after 4 and 8 weeks of healing. The TiZr-H group revealed significant differences $(p<0.05)$ to the TiZr group after 4 weeks between 1.14 and 1.19 mm. After 8 weeks of healing, the TiZr-Sr group exhibited significant differences to the TiZr-EMD group in the ranges of $0.40-0.60 \mathrm{~mm}$ and $1.11-2.08 \mathrm{~mm}(n=4)$.

ment of all markers, except from BMD that remained almost unchanged for all groups. By contrast, the trend for TiZr-H and TiZr-Sr groups was toward decreasing values, while TiZr group only exhibited marginally positive changes.

We also evaluated the 2D BMD of tissues within the ROI to investigate the bone healing by increasing layers after 4 and 8 weeks of healing (Figure 5A and B). Plotting of the depth profiles for $2 \mathrm{D} \mathrm{BMD}$ could also represent the release and diffusion rates of EMD and strontium in vivo. The results indicated significant differences of TiZr-H to TiZr group after 4 weeks of healing, between $1.14 \mathrm{~mm}$ and $1.19 \mathrm{~mm}$. TiZr-Sr group exhibited significant differences to TiZr-EMD group after 8 weeks of healing in the ranges of $0.40-0.60 \mathrm{~mm}$ and 1.11-2.08 $\mathrm{mm}$, indicating more EMD release and diffusion toward the central bone defect. However, after 4 weeks of healing, we observed higher diffusion rates by increasing depth for strontium than EMD.

3.4. Histologic and Histolmorphometric Analyses. Regarding implants' biocompatibility and bone healing properties, there was no significant difference between coated and uncoated groups at both time points. Figure $6 \mathrm{~A}$ and $\mathrm{B}$ shows that strontium and EMD coating layers could positively affect the bone healing potential of TiZr implants. Using $p<0.05$, the normality test (Shapiro-Wilk) failed for both indexes. We also calculated the horizontal (index A) and vertical (index C) dimensions of regenerated bone at both time points (Figure 6C). Although no statistically significant difference was observed for both index $\mathrm{A}$ and $\mathrm{C}$ between groups, strontium had better healing effects in both dimensions after 4 weeks of healing. In addition, EMD induced more bone healing after 8 weeks of healing.

\section{DISCUSSION}

Even though many types of biomolecules are suggested for surface functionalization, only few studies compared the efficacy of different modifications with each other in the same experimental model. ${ }^{39-41}$ To the best of our knowledge, no studies compared the osteoimmunomodulatory potential of surface functionalization using EMD with nanostructure surface modification by strontium ions. The mentioned difficulties with using EMD inspired us to investigate whether strontium ions could be considered as a simpler, safer, and cheaper alternative for inducing bone healing and modulating the immune system responses.

The results indicated that strontium induced higher bone surface to volume ratio, BMD, and bone surface/tissue volume after 4 weeks of healing compared to EMD. However, EMD 

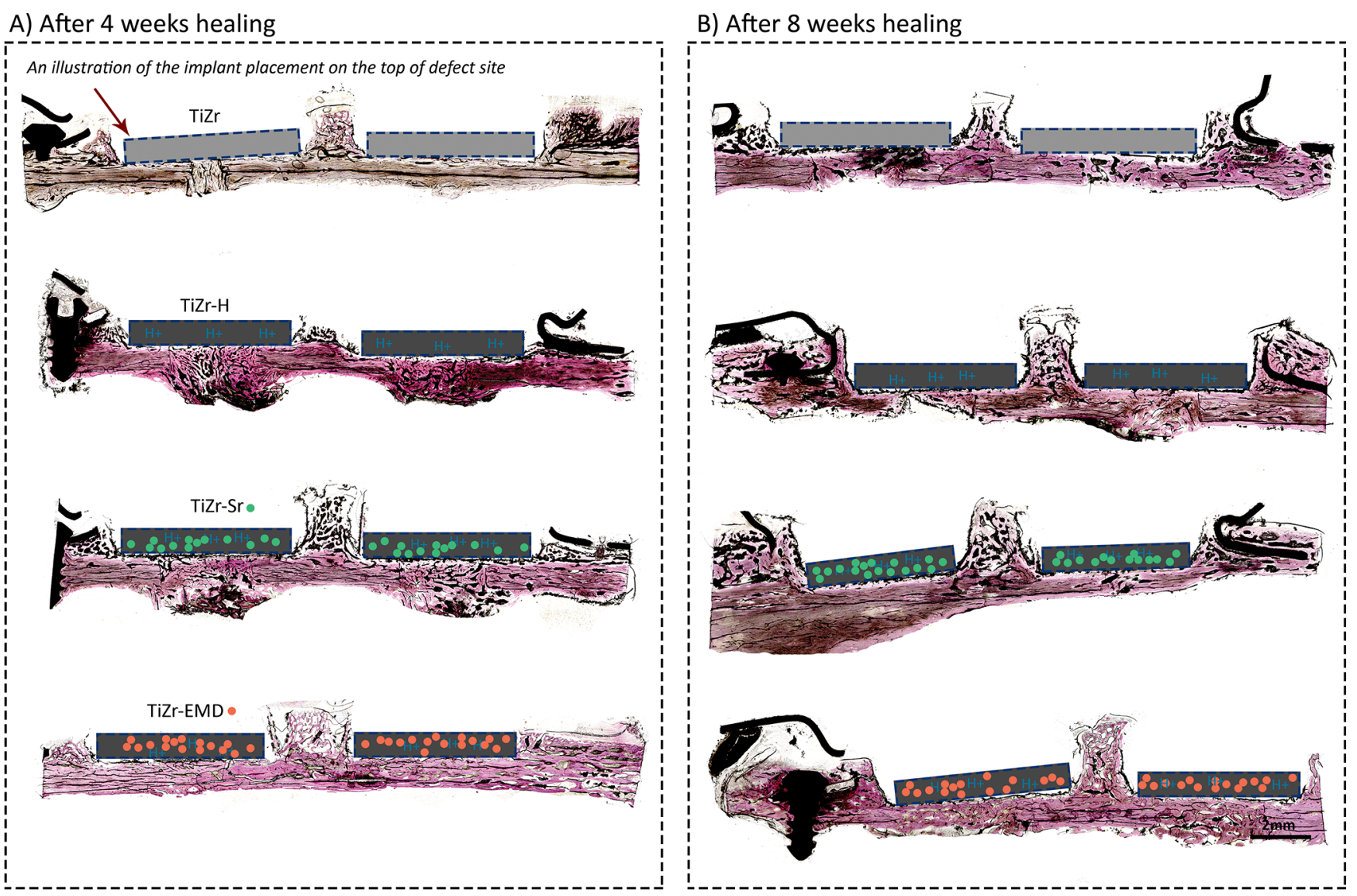

C) Histomorphometry

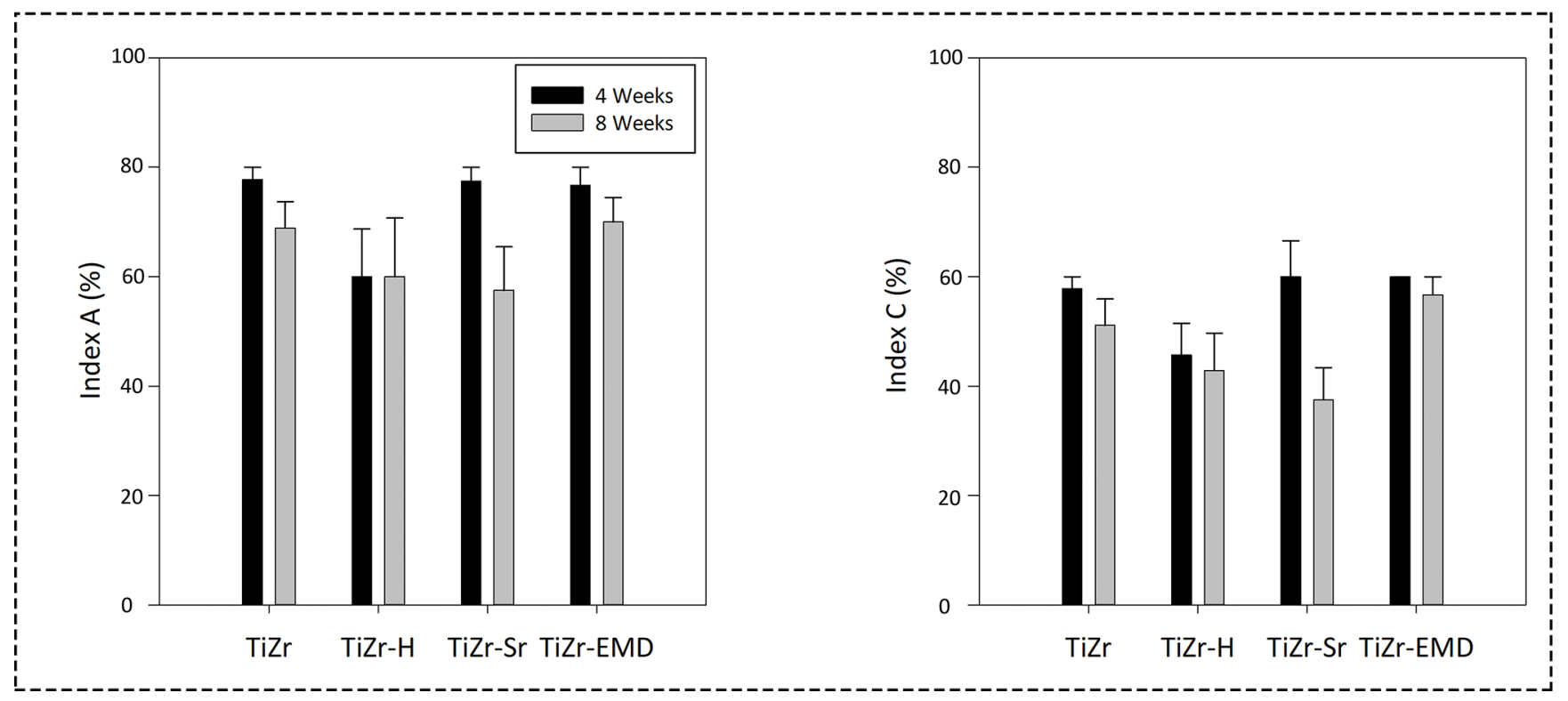

Figure 6. Representative images of H\&E staining and histomorphometry results after 4 and 8 weeks of healing. H\&E staining of all groups 4 (A) and 8 (B) weeks after implantation in 10× magnification. The green and orange dots are the illustrations of strontium and EMD, respectively. (C) Histomorphometric analysis of regenerated bone in horizontal (index A) and vertical (index C) dimensions $(n=4)$.

appeared to affect the late inflammatory responses, when comparing the differences between 4 and 8 weeks of healing. This was in agreement with other studies that reported the long-term bone healing effects of EMD. ${ }^{56}$ A titanium surface modified with peptides or protein domains with Arg-Gly-Asp (RGD) motifs, like amelogenin, the main component in EMD, facilitates the cell adhesion and signal transduction with positive effects on the differentiation of osteoblasts. ${ }^{57}$ The observed short-term bone healing effects of strontium were also in agreement with the Lin et al. ${ }^{50}$ study, which demonstrated that strontium-modified surfaces induce early osseointegration in rabbits. $^{50}$ 
The 2D micro-CT analysis revealed an inhomogeneous distribution of BMD in relation to the implant surface distance in all groups. The micro-CT analysis also presented that the $\mathrm{BMD}$ in the plane close to the implant surface was almost twice higher than the average BMD for the measured defect. The BMD results in the plane close to the implant surface indicated higher strontium amounts by increasing depth after 4 weeks than those of EMD. This could indicate that the nanostructured modified surface with strontium ions induced more inflammation and fibrin clots representing the early phase of wound healing, whereas EMD induce the later cellular phase with cellular remodeling. ${ }^{58}$ This was confirmed by the lower cytotoxicity and inflammatory markers expression in TiZr-EMD group compared to other groups. In contrast, the ALP activity in the wound fluid was higher in this group compared to both TiZr-H and TiZr-Sr groups after 8 weeks of healing. These data could be a support for osteoimmunomodulatory effects of EMD coating layer on implants after implantation.

Our in vitro studies on MC3T3-E1 cells exhibited higher Coll-1 gene expression for EMD-coated implants than uncoated control group. ${ }^{42}$ Likewise, Reseland et al. ${ }^{59}$ incubated primary human osteoblasts with EMD and their results indicated increasing levels of Coll-1 gene expression and IL-6 secretion for up to 7 days. ${ }^{59}$ Nevertheless, because of the complex physiologic conditions at the body with different types of cells, we could not compare the results of basic in vitro cell cultures with animal study outcomes. The faster bone growth in rabbits than humans and the prolonged time points used in this study may also be other reasons for the different findings. In addition, Miron et al. ${ }^{60}$ coated EMD on a titanium SLA surface (Straumann, Basel, Switzerland) by a dipping procedure and then studied the osteoblasts responses in vitro. ${ }^{60}$ Their results indicated that EMD accelerated the differentiation of calvarial osteoblasts by promoting mature phenotypes earlier than surfaces without EMD. ${ }^{60}$ While strontium induced ALP gene activity after 4 weeks, TiZr and TiZr-EMD groups were the only groups with increased $A L P$ gene activity after 8 weeks of healing. We also observed a clear trend toward lower inflammation markers in TiZr-EMD group, which indicated the positive effect of EMD on suppressing inflammation and modulating immune system responses. Tissue on the sr-coated implants had a lower TRAP mRNA levels after 8 weeks as compared to uncoated control and TiZH-EMD groups. Park et al. ${ }^{61}$ coated a $\mathrm{srTiO}_{3}$ layer on screw shaped titanium implants with a moderately rough surface and studied the gene expression levels after 2 weeks implantation in rabbits. ${ }^{61}$ The authors reported significantly higher OC, TNF- $\alpha$, and $I L-10$ gene levels but lower TRAP gene expression levels. $^{61}$ Likewise, Zhao et al. ${ }^{62}$ reported significantly higher $O C$, Coll-1, and $A L P$ gene expression in an in vitro study with rat cells for strontium-modified titanium nanotubes compared to uncoated groups after 2 weeks. ${ }^{62}$

Regarding the bone regeneration potential of implants, the histologic and histomorphometric analyses showed no significant differences between groups. Besides, we observed no significant difference after functionalization of implant surface using cathodic polarization. Even though the results of this study could support the EMD long-term immunomodulatory effects, the selected time points might have been too prolonged to assess a clearer impact of the individual functionalized surfaces on bone formation.
The animal model and implant shape used in this study are recognized as validated models for evaluating dental implants, and the details have been discussed in our previous papers. ${ }^{48,63-65}$ Studying the bone healing effects of these biomolecules in animal models for systematic disorders or poor bone quality could provide more information. Implants with combined surface modifications could have identified if these treatments induce additive or synergistic effects; however, the aim of this study was to test the two different implant modifications individually to compare their molecular and biological effects.

\section{CONCLUSIONS}

The purpose of this study was to compare the osteoimmunomodulatory effects of ion deposition and biomolecule functionalization on implant surface in vivo. Regarding the bone healing potential of implants, our histologic data showed no significant difference between groups after 4 and 8 weeks healing in vivo. Biofunctionalization with EMD could modulate the later immune system responses to implants. Based on the present study, strontium ion deposition on implant surface could not provide the similar osteoimmunomodulatory effects of EMD surface biofunctionalization. Since we used rabbit animals with no systematic disorders or poor bone quality in this study, we could not detect any significant difference between groups under bone inefficiency conditions. Studying the bone healing effects of these treatments in animal models for systematic disorders or poor bone quality might provide more information.

\section{ASSOCIATED CONTENT}

\section{(s) Supporting Information}

The Supporting Information is available free of charge at https://pubs.acs.org/doi/10.1021/acsabm.0c00608.

Video S1. Coating a layer of strontium and EMD on titanium-based implants using electrochemical cathodic polarization method (AVI)

Figure S1. Dot blot quantification of EMD released from TiZr-EMD implants (PDF)

\section{AUTHOR INFORMATION}

\section{Corresponding Author}

Håvard Jostein Haugen - Department of Biomaterials, Institute for Clinical Dentistry, University of Oslo, NO-0317 Oslo, Norway; (1) orcid.org/0000-0002-6690-7233; Email: h.j.haugen@odont.uio.no

\section{Authors}

Maryam Rahmati - Department of Biomaterials, Institute for Clinical Dentistry, University of Oslo, NO-0317 Oslo, Norway

Matthias Johannes Frank - Department of Biomaterials, Institute for Clinical Dentistry, University of Oslo, NO-0317 Oslo, Norway; Institute of Medical and Polymer Engineering, Chair of Medical Engineering, Technische Universität München, 85748 Garching, Germany

Sebastian Martin Walter - Department of Biomaterials, Institute for Clinical Dentistry, University of Oslo, NO-0317 Oslo, Norway; Institute of Medical and Polymer Engineering, Chair of Medical Engineering, Technische Universität München, 85748 Garching, Germany

Marta Cabrer Monjo - Department of Fundamental Biology and Health Sciences, Research Institute on Health Sciences 
(IUNICS), University of Balearic Islands, ES-07122 Palma, Spain; Balearic Islands Health Institute (IdISBa), ES-07010 Palma, Spain

Maria Satue - Department of Fundamental Biology and Health Sciences, Research Institute on Health Sciences (IUNICS), University of Balearic Islands, ES-07122 Palma, Spain; Balearic Islands Health Institute (IdISBa), ES-07010 Palma, Spain; Department of Biomedical Sciences, University of Veterinary Medicine, 1210 Vienna, Austria

Janne Elin Reseland - Department of Biomaterials, Institute for Clinical Dentistry, University of Oslo, NO-0317 Oslo, Norway

Ståle Petter Lyngstadaas - Department of Biomaterials, Institute for Clinical Dentistry, University of Oslo, NO-0317 Oslo, Norway

Complete contact information is available at: https://pubs.acs.org/10.1021/acsabm.0c00608

\section{Notes}

The authors declare no competing financial interest.

\section{ACKNOWLEDGMENTS}

This work was supported by a project "Promoting patient safety by a novel combination of imaging technologies for biodegradable magnesium implants, MgSafe" funded by European Training Network within the framework of Horizon 2020 Marie Skłodowska-Curie Action (MSCA) grant number No 811226 (www.mgsafe.eu) and Research Council of Norway grant no. 231530. We kindly acknowledge Ingrid Andersbakken, Heidi Straume Haugland, Hans Jacob Rønold, and Jan Eirik Ellingsen for their assistance during the animal surgery and implants characterizations. Institut Straumann AG, Basel, Switzerland, is acknowledged for providing the TiZr SLActive coins.

\section{REFERENCES}

(1) Stewart, C.; Akhavan, B.; Wise, S. G.; Bilek, M. M. M. A review of biomimetic surface functionalization for bone-integrating orthopedic implants: Mechanisms, current approaches, and future directions. Prog. Mater. Sci. 2019, 106, 100588.

(2) Shuai, C. J.; Li, S.; Peng, S. P.; Feng, P.; Lai, Y. X.; Gao, C. D. Biodegradable metallic bone implants. Materials Chemistry Frontiers 2019, 3 (4), 544-562.

(3) Goodman, S. B.; Yao, Z.; Keeney, M.; Yang, F. The future of biologic coatings for orthopaedic implants. Biomaterials 2013, 34 (13), 3174-83.

(4) Xing, R.; Lyngstadaas, S. P.; Ellingsen, J. E.; Taxt-Lamolle, S.; Haugen, H. J. The influence of surface nanoroughness, texture and chemistry of TiZr implant abutment on oral biofilm accumulation. Clin Oral Implants Res. 2015, 26 (6), 649-56.

(5) Chandorkar, Y.; K, R.; Basu, B. The foreign body response demystified. ACS Biomater. Sci. Eng. 2019, 5 (1), 19-44.

(6) Magill, S. S.; Edwards, J. R.; Bamberg, W.; Beldavs, Z. G.; Dumyati, G.; Kainer, M. A.; Lynfield, R.; Maloney, M.; McAllisterHollod, L.; Nadle, J.; Ray, S. M.; Thompson, D. L.; Wilson, L. E.; Fridkin, S. K. Emerging Infections Program Healthcare-Associated, I.; Antimicrobial Use Prevalence Survey, T., Multistate point-prevalence survey of health care-associated infections. N. Engl. J. Med. 2014, 370 (13), 1198-208.

(7) Sikora, C. L.; Alfaro, M. F.; Yuan, J. C.; Barao, V. A.; Sukotjo, C.; Mathew, M. T. Wear and Corrosion Interactions at the Titanium/ Zirconia Interface: Dental Implant Application. J. Prosthodontics 2018, 27 (9), 842-852.

(8) Ghazal, S. S.; Huynh-Ba, G.; Aghaloo, T.; Dibart, S.; Froum, S.; O’Neal, R.; Cochran, D. A Randomized, Controlled, Multicenter Clinical Study Evaluating The Crestal Bone Level Change Of
SLActive Bone Level O $3.3 \mathrm{~mm}$ Implants Compared To SLActive Bone Level O $4.1 \mathrm{~mm}$ Implants For Single-Tooth Replacement. Int. J. Oral Maxillofac Implants 2019, 34 (3), 708-718.

(9) Frank, M. J.; Walter, M. S.; Lyngstadaas, S. P.; Wintermantel, E.; Haugen, H. J. Hydrogen content in titanium and a titanium-zirconium alloy after acid etching. Mater. Sci. Eng., C 2013, 33 (3), 1282-8.

(10) Zheng, X.-f.; Mo, A.-c., Immune System-Related Diseases and Dental Implant Treatment. In Dental Implant Treatment in Medically Compromised Patients; Springer: 2020; pp 129-147.

(11) Chen, H.; Liu, N.; Xu, X.; Qu, X.; Lu, E. Smoking, radiotherapy, diabetes and osteoporosis as risk factors for dental implant failure: a meta-analysis. PLoS One 2013, 8 (8), e71955.

(12) Chen, W.; Shen, X.; Hu, Y.; Xu, K.; Ran, Q.; Yu, Y.; Dai, L.; Yuan, Z.; Huang, L.; Shen, T.; Cai, K. Surface functionalization of titanium implants with chitosan-catechol conjugate for suppression of ROS-induced cells damage and improvement of osteogenesis. Biomaterials 2017, 114, 82-96.

(13) Jurczak, P.; Witkowska, J.; Rodziewicz-Motowidlo, S.; Lach, S. Proteins, peptides and peptidomimetics as active agents in implant surface functionalization. Adv. Colloid Interface Sci. 2020, 276, 102083.

(14) Walter, M. S.; Frank, M. J.; Satue, M.; Monjo, M.; Ronold, H. J.; Lyngstadaas, S. P.; Haugen, H. J. Bioactive implant surface with electrochemically bound doxycycline promotes bone formation markers in vitro and in vivo. Dent. Mater. 2014, 30 (2), 200-14.

(15) Yu, Q.; Zhang, Y.; Wang, H.; Brash, J.; Chen, H. Anti-fouling bioactive surfaces. Acta Biomater. 2011, 7 (4), 1550-7.

(16) Raphel, J.; Karlsson, J.; Galli, S.; Wennerberg, A.; Lindsay, C.; Haugh, M. G.; Pajarinen, J.; Goodman, S. B.; Jimbo, R.; Andersson, M.; Heilshorn, S. C. Engineered protein coatings to improve the osseointegration of dental and orthopaedic implants. Biomaterials 2016, 83, 269-82.

(17) Bahna, P.; Dvorak, T.; Hanna, H.; Yasko, A. W.; Hachem, R.; Raad, I. Orthopaedic metal devices coated with a novel antiseptic dye for the prevention of bacterial infections. Int. J. Antimicrob. Agents 2007, 29 (5), 593-6.

(18) Kim, J.; Kim, S. K.; Heo, S. J.; Koak, J. Y. Bone Formation of Titanium Implant Surfaces Treated with Submicron Poly(lactide-coglycolide)/Recombinant Human Transforming Growth Factor- beta2 Particles by the Electrospray Method: An In Vivo Study. Int. J. Oral Maxillofac Implants 2019, 34 (6), 1404-1412.

(19) O’Neill, E.; Awale, G.; Daneshmandi, L.; Umerah, O.; Lo, K. W. The roles of ions on bone regeneration. Drug Discovery Today 2018, 23 (4), 879-890.

(20) Dai, Y.; Guo, H.; Chu, L.; He, Z.; Wang, M.; Zhang, S.; Shang, $X$. Promoting osteoblasts responses in vitro and improving osteointegration in vivo through bioactive coating of nanosilicon nitride on polyetheretherketone. J. Ortho. Trans. 2019, DOI: $10.1016 /$ j.jot.2019.10.011.

(21) Tsukanaka, M.; Fujibayashi, S.; Takemoto, M.; Matsushita, T.; Kokubo, T.; Nakamura, T.; Sasaki, K.; Matsuda, S. Bioactive treatment promotes osteoblast differentiation on titanium materials fabricated by selective laser melting technology. Dent. Mater. J. 2016, 35 (1), 118-25.

(22) Izquierdo-Barba, I.; Santos-Ruiz, L.; Becerra, J.; Feito, M. J.; Fernandez-Villa, D.; Serrano, M. C.; Diaz-Guemes, I.; FernandezTome, B.; Enciso, S.; Sanchez-Margallo, F. M.; Monopoli, D.; Afonso, H.; Portoles, M. T.; Arcos, D.; Vallet-Regi, M. Synergistic effect of Sihydroxyapatite coating and VEGF adsorption on Ti6Al4V-ELI scaffolds for bone regeneration in an osteoporotic bone environment. Acta Biomater. 2019, 83, 456-466.

(23) Lo, K. W.; Ashe, K. M.; Kan, H. M.; Laurencin, C. T. The role of small molecules in musculoskeletal regeneration. Regener. Med. 2012, 7 (4), 535-49.

(24) Wang, L.; Yang, X.; Cao, W. W.; Shi, C.; Zhou, P. H.; Li, Q.; Han, F. X.; Sun, J. Y.; Xing, X. D.; Li, B. Mussel-inspired deposition of copper on titanium for bacterial inhibition and enhanced osseointegration in a periprosthetic infection model. RSC $A d v .2017,7$ (81), 51593-51604. 
(25) Smeets, R.; Precht, C.; Hahn, M.; Jung, O.; Hartjen, P.; Heiland, M.; Grobe, A.; Holthaus, M. G.; Hanken, H. Biocompatibility and Osseointegration of Titanium Implants with a Silver-Doped Polysiloxane Coating: An In Vivo Pig Model. Int. J. Oral Maxillofac Implants 2017, 32 (6), 1338-1345.

(26) Tao, Z. S.; Zhou, W. S.; He, X. W.; Liu, W.; Bai, B. L.; Zhou, Q.; Huang, Z. L.; Tu, K. K.; Li, H.; Sun, T.; Lv, Y. X.; Cui, W.; Yang, L. A comparative study of zinc, magnesium, strontium-incorporated hydroxyapatite-coated titanium implants for osseointegration of osteopenic rats. Mater. Sci. Eng., C 2016, 62, 226-32.

(27) Alenezi, A.; Galli, S.; Atefyekta, S.; Andersson, M.; Wennerberg, A. Osseointegration effects of local release of strontium ranelate from implant surfaces in rats. J. Mater. Sci.: Mater. Med. 2019, 30 (10), 116.

(28) Marie, P. J.; Felsenberg, D.; Brandi, M. L. How strontium ranelate, via opposite effects on bone resorption and formation, prevents osteoporosis. Osteoporosis Int. 2011, 22 (6), 1659-67.

(29) Rahmati, M.; Mozafari, M. Selective Contribution of Bioactive Glasses to Molecular and Cellular Pathways. ACS Biomater. Sci. Eng. 2020, 6 (1), 4-20.

(30) Miron, R. J.; Sculean, A.; Cochran, D. L.; Froum, S.; Zucchelli, G.; Nemcovsky, C.; Donos, N.; Lyngstadaas, S. P.; Deschner, J.; Dard, M.; Stavropoulos, A.; Zhang, Y.; Trombelli, L.; Kasaj, A.; Shirakata, Y.; Cortellini, P.; Tonetti, M.; Rasperini, G.; Jepsen, S.; Bosshardt, D. $D$. Twenty years of enamel matrix derivative: the past, the present and the future. J. Clin. Periodontol. 2016, 43 (8), 668-83.

(31) Takeda, K.; Mizutani, K.; Matsuura, T.; Kido, D.; Mikami, R.; Noda, M.; Buranasin, P.; Sasaki, Y.; Izumi, Y. Periodontal regenerative effect of enamel matrix derivative in diabetes. PLoS One 2018, 13 (11), No. e0207201.

(32) Kato, H.; Taguchi, Y.; Imai, K.; Ruan, Y.; Tsai, Y.-W.; Chen, Y.C.; Shida, M.; Taguchi, R.; Tominaga, K.; Umeda, M. The Effects of Amelogenin Exon 5 Encoded Peptide from Enamel Matrix Derivative Enhances in Odontoblast like Cells, KN-3 Cells. 2018. Appl. Sci. 2018, 8 (10), 1890.

(33) Song, Z. C.; Li, S.; Dong, J. C.; Sun, M. J.; Zhang, X. L.; Shu, R. Enamel matrix proteins regulate hypoxia-induced cellular biobehavior and osteogenic differentiation in human periodontal ligament cells. Biotech. Histochem. 2017, 92 (8), 606-618.

(34) Park, J. S.; Pabst, A. M.; Ackermann, M.; Moergel, M.; Jung, J.; Kasaj, A. Biofunctionalization of porcine-derived collagen matrix using enamel matrix derivative and platelet-rich fibrin: influence on mature endothelial cell characteristics in vitro. Clin Oral Investig 2018, 22 (2), 909-917.

(35) Pullisaar, H.; Verket, A.; Szoke, K.; Tiainen, H.; Haugen, H. J.; Brinchmann, J. E.; Reseland, J. E.; Ostrup, E. Alginate hydrogel enriched with enamel matrix derivative to target osteogenic cell differentiation in $\mathrm{TiO} 2$ scaffolds. J. Tissue Eng. 2015, 6, 204173141557587.

(36) Villa, O.; Wohlfahrt, J. C.; Mdla, I.; Petzold, C.; Reseland, J. E.; Snead, M. L.; Lyngstadaas, S. P. Proline-Rich Peptide Mimics Effects of Enamel Matrix Derivative on Rat Oral Mucosa Incisional Wound Healing. J. Periodontol. 2015, 86 (12), 1386-1395.

(37) Al-Dabbagh, N. N.; Zahid, T. M. The impact of fatwas on patients' acceptance of enamel matrix derivatives for periodontal regeneration in Saudi Arabia. Patient Prefer Adherence 2018, 12, 2405-2411

(38) Ramis, J. M.; Rubert, M.; Vondrasek, J.; Gaya, A.; Lyngstadaas, S. P.; Monjo, M. Effect of enamel matrix derivative and of proline-rich synthetic peptides on the differentiation of human mesenchymal stem cells toward the osteogenic lineage. Tissue Eng., Part A 2012, 18 (1112), 1253-63.

(39) Civantos, A.; Martinez-Campos, E.; Ramos, V.; Elvira, C.; Gallardo, A.; Abarrategi, A. Titanium Coatings and Surface Modifications: Toward Clinically Useful Bioactive Implants. ACS Biomater. Sci. Eng. 2017, 3 (7), 1245-1261.

(40) Todea, M.; Vulpoi, A.; Popa, C.; Berce, P.; Simon, S. Effect of different surface treatments on bioactivity of porous titanium implants. J. Mater. Sci. Technol. 2019, 35 (3), 418-426.
(41) Meng, H. W.; Chien, E. Y.; Chien, H. H. Dental implant bioactive surface modifications and their effects on osseointegration: a review. Biomark Res. 2016, 4 (1), 24.

(42) Frank, M. J.; Walter, M. S.; Rubert, M.; Thiede, B.; Monjo, M.; Reseland, J. E.; Haugen, H. J.; Lyngstadaas, S. P. Cathodic Polarization Coats Titanium Based Implant Materials with Enamel Matrix Derivate (EMD). Materials 2014, 7 (3), 2210-2228.

(43) Frank, M. J.; Walter, M. S.; Tiainen, H.; Rubert, M.; Monjo, M.; Lyngstadaas, S. P.; Haugen, H. J. Coating of metal implant materials with strontium. J. Mater. Sci.: Mater. Med. 2013, 24 (11), 2537-48.

(44) Alcazar, J. C.; Salas, M. M.; Conde, M. C.; Chisini, L. A.; Demarco, F. F.; Tarquinio, S. B.; Carreno, N. L. Electrochemical Cathodic Polarization, a Simplified Method That Can. Modified and Increase the Biological Activity of Titanium Surfaces: A Systematic Review. PLoS One 2016, 11 (7), No. e0155231.

(45) Alves, A. C.; Wenger, F.; Ponthiaux, P.; Celis, J. P.; Pinto, A. M.; Rocha, L. A.; Fernandes, J. C. S. Corrosion mechanisms in titanium oxide-based films produced by anodic treatment. Electrochim. Acta 2017, 234, 16-27.

(46) Pachauri, P.; Bathala, L. R.; Sangur, R. Techniques for dental implant nanosurface modifications. J. Adv. Prosthodont 2014, 6 (6), 498-504.

(47) Szmukler-Moncler, S.; Bischof, M.; Nedir, R.; Ermrich, M. Titanium hydride and hydrogen concentration in acid-etched commercially pure titanium and titanium alloy implants: a comparative analysis of five implant systems. Clin. Oral Implants Res. 2010, 21 (9), 944-950.

(48) Rahmati, M.; Lyngstadaas, S. P.; Reseland, J. E.; Andersbakken, I.; Haugland, H. S.; López-Peña, M.; Cantalapiedra, A. G.; Muñoz, F. M. G.; Haugen, H. J. Coating doxycycline on titanium-based implants: Two in vivo studies. Bioactive Materials 2020, 5 (4), 787-797.

(49) Wohlfahrt, J. C.; Monjo, M.; Ronold, H. J.; Aass, A. M.; Ellingsen, J. E.; Lyngstadaas, S. P. Porous titanium granules promote bone healing and growth in rabbit tibia peri-implant osseous defects. Clin Oral Implants Res. 2010, 21 (2), 165-73.

(50) Lin, G.; Zhou, C.; Lin, M.; Xu, A.; He, F. Strontiumincorporated titanium implant surface treated by hydrothermal reactions promotes early bone osseointegration in osteoporotic rabbits. Clin Oral Implants Res. 2019, 30 (8), 777-790.

(51) Rønold, H. J.; Ellingsen, J. E. The use of a coin shaped implant for direct in situ measurement of attachment strength for osseointegrating biomaterial surfaces. Biomaterials 2002, 23 (10), 2201-2209.

(52) Rønold, H. J.; Ellingsen, J. E.; Lyngstadaas, S. P. Tensile force testing of optimized coin-shaped titanium implant attachment kinetics in the rabbit tibiae. J. Mater. Sci.: Mater. Med. 2003, 14 (10), 843849.

(53) Monjo, M.; Lamolle, S. F.; Lyngstadaas, S. P.; Ronold, H. J.; Ellingsen, J. E. In vivo expression of osteogenic markers and bone mineral density at the surface of fluoride-modified titanium implants. Biomaterials 2008, 29 (28), 3771-80.

(54) Monjo, M.; Rubert, M.; Wohlfahrt, J. C.; Ronold, H. J.; Ellingsen, J. E.; Lyngstadaas, S. P. In vivo performance of absorbable collagen sponges with rosuvastatin in critical-size cortical bone defects. Acta Biomater. 2010, 6 (4), 1405-12.

(55) Dalby, M. J.; Gadegaard, N.; Tare, R.; Andar, A.; Riehle, M. O.; Herzyk, P.; Wilkinson, C. D.; Oreffo, R. O. The control of human mesenchymal cell differentiation using nanoscale symmetry and disorder. Nat. Mater. 2007, 6 (12), 997.

(56) Rodrigues, T. L.; Marchesan, J. T.; Coletta, R. D.; Novaes, A. B., Jr.; Grisi, M. F.; Souza, S. L.; Taba, M., Jr.; Palioto, D. B. Effects of enamel matrix derivative and transforming growth factor-betal on human periodontal ligament fibroblasts. J. Clin. Periodontol. 2007, 34 (6), 514-22.

(57) Schliephake, H.; Scharnweber, D.; Dard, M.; Sewing, A.; Aref, A.; Roessler, S. Functionalization of dental implant surfaces using 
adhesion molecules. J. Biomed. Mater. Res., Part B 2005, 73 (1), 8896.

(58) Villar, C. C.; Huynh-Ba, G.; Mills, M. P.; Cochran, D. L. Wound healing around dental implants. Endodontic Topics 2011, 25 (1), 44-62.

(59) Reseland, J. E.; Reppe, S.; Larsen, A. M.; Berner, H. S.; Reinholt, F. P.; Gautvik, K. M.; Slaby, I.; Lyngstadaas, S. P. The effect of enamel matrix derivative on gene expression in osteoblasts. Eur. J. Oral Sci. 2006, 114 (Suppl 1), 205-11 discussion 254-6, 381-2..

(60) Miron, R. J.; Oates, C. J.; Molenberg, A.; Dard, M.; Hamilton, D. W. The effect of enamel matrix proteins on the spreading, proliferation and differentiation of osteoblasts cultured on titanium surfaces. Biomaterials 2010, 31 (3), 449-60.

(61) Park, J. W.; Kim, Y. J.; Jang, J. H.; Song, H. Positive modulation of osteogenesis- and osteoclastogenesis-related gene expression with strontium-containing microstructured Ti implants in rabbit cancellous bone. J. Biomed. Mater. Res., Part A 2013, 101 (1), 298-306.

(62) Zhao, L.; Wang, H.; Huo, K.; Zhang, X.; Wang, W.; Zhang, Y.; $\mathrm{Wu}, \mathrm{Z}$.; Chu, P. K. The osteogenic activity of strontium loaded titania nanotube arrays on titanium substrates. Biomaterials 2013, 34 (1), $19-29$.

(63) Wohlfahrt, J. C.; Monjo, M.; Rønold, H. J.; Aass, A. M.; Ellingsen, J. E.; Lyngstadaas, S. P. Porous titanium granules promote bone healing and growth in rabbit tibia peri-implant osseous defects. Clinical oral implants research 2010, 21 (2), 165-173.

(64) Stübinger, S.; Dard, M. The rabbit as experimental model for research in implant dentistry and related tissue regeneration. Journal of Investigative Surgery 2013, 26 (5), 266-282.

(65) Godoy-Gallardo, M.; Manzanares-Céspedes, M. C.; Sevilla, P.; Nart, J.; Manzanares, N.; Manero, J. M.; Gil, F. J.; Boyd, S. K.; Rodríguez, D. Evaluation of bone loss in antibacterial coated dental implants: An experimental study in dogs. Mater. Sci. Eng., C 2016, 69, 538-545. 